\title{
Concurrent Operation of Two Over-the-Horizon Radars
}

\author{
Yimin Zhang, Senior Member, IEEE, Gordon J. Frazer, Member, IEEE, and Moeness G. Amin, Fellow, IEEE
}

\begin{abstract}
By exploiting the reflective and refractive nature of high-frequency (HF) radiowave propagation through the ionosphere or the conducting sea surface, over-the-horizon radar (OTHR) systems perform wide-area surveillance at long range well beyond the limit of the horizon of conventional line-of-sight (LOS) radars. Improved characterizations of the targets can be achieved by using multiple OTHRs operating simultaneously as compared to a single OTHR operating alone. In this paper, we consider concurrent operations of two OTHR systems that occupy the same frequency band with different chirp waveforms. The objective is to respond to the advanced wide-area surveillance needs without reducing the wave repetitive frequency. For this purpose, a new cross-radar interference cancellation technique is developed and its effectiveness is verified through both analytical and simulation results.
\end{abstract}

\section{INTRODUCTION}

O VER-THE-HORIZON RADAR (OTHR) systems perform wide-area surveillance at long range well beyond the limit of the horizon of conventional line-of-sight (LOS) radars [1]-[3]. It can track aircraft more than $3000 \mathrm{~km}$ away and over millions of square kilometers of open ocean [4]. Further, OTHR permits more accurate landfalls of Hurricane landfall, with more complete information about the size, shape, and extent of the interface between storm and ambient airflow. In coastal wave forecasts, OTHR can characterize the wave field in the open ocean from which coastal forecasts are derived, so as early evacuations of coastal areas.

With a single OTHR, information about the target range and Doppler frequency in the slant range direction can be obtained. However, such information does not uniquely determine the movement of the targets. To respond to the needs for advanced wide-area surveillance, we propose in this paper a concurrent operation of two OTHR systems. The use of two OTHRs, positioned at different locations, not only extends the coverage for enhanced surveillance, but also offers higher-dimensional information of a moving target. This information is key in achieving

Manuscript received September 1, 2006; revised February 14, 2007. This work was supported in part by the Office of Naval Research under Grant N00014-98-1-0176 and the ONR/NSWC under Contract N65540-05-C-0028. The associate editor coordinating the review of this manuscript and approving it for publication was Prof. Antonia Papandreou-Suppappola.

Y. Zhang and M. G. Amin are with the Center for Advanced Communications, Villanova University, Villanova, PA 19085 USA (e-mail: yimin@ ieee.org; moeness.amin@ villanova.edu).

G. J. Frazer is with Intelligence, Surveillance and Reconnaissance Division, Defence Science and Technology Organisation, Edinburgh, SA 5111, Australia (e-mail: frazer@ieee.org).

Digital Object Identifier 10.1109/JSTSP.2007.897054

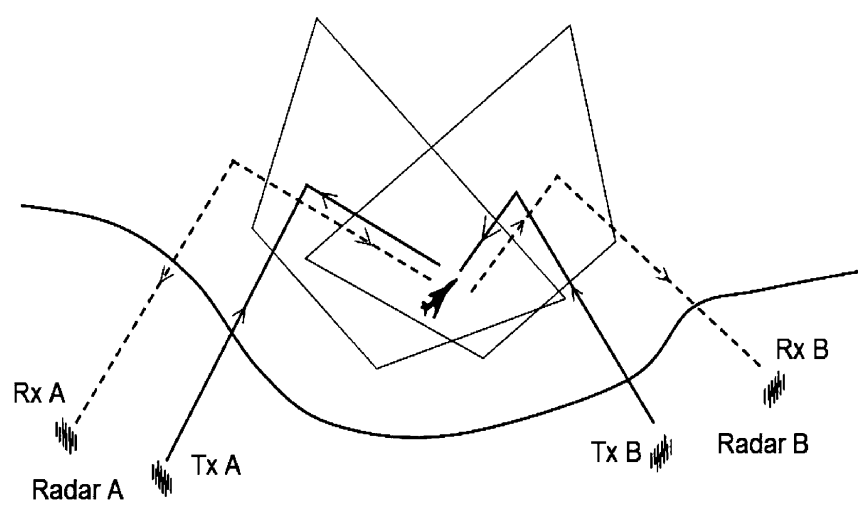

Fig. 1. Illustration of concurrent operation of multiple OTHRs.

improved target classification and predictions of ballistic destinations. Fig. 1 illustrates such a scenario. When only radar A is operational, the range and Doppler information is estimated in terms of (Tx A - target - Rx A). With radar B added to the operation, information about the following combinations can also be obtained: (Tx B - target - Rx B, Tx A - target - Rx B, Tx B target $-\mathrm{Rx} \mathrm{A}$ ). In a single-radar setting, the movement of a target in the direction orthogonal to the slant path between radar A and the target can only be detected when the target passes through different cross-range bins. In a dual-radar system, on the other hand, Doppler frequencies related to the aforementioned four slant path combinations can be detected when radar B is also employed as the transmitter or/and receiver.

A key limitation of HF radar is the tradeoff between the selection of an appropriate operating frequency and the demand for radar waveform bandwidth that is commensurate with the radar range resolution requirements. While the operation band of OTHR systems is nominally 3-30 MHz, the effective operating frequency bandwidth available to a particular radar at any given time is further limited due to propagation constraints. The problem described above is further compounded when a network of two or more radars is in use. Present operation of multiple OTHR systems requires the reduction of wave repetition frequency (WRF) or the division of the waveform bandwidth. These conditions can be relaxed if effective mitigation of cross-radar interference can be achieved [5]. In this case, the same frequency band can be concurrently occupied by two OTHR systems with different waveforms, leaving both the WRF and bandwidth uncompromised.

In this paper, we consider linear frequency modulated (LFM) waveforms with different frequency sweeping orientations. In general, modern OTHRs use LFM pulses (for mono-static radar designs) or linear frequency-modulated continuous-wave (FM/CW) waveforms for bistatic designs (typical of the larger 
skywave radar cases). While discrete coded waveforms are used in some radar systems, they are not the preferred signal mode of operation in OTHR [5]. LFM (indeed almost any waveform with continuous and differentiable phase law) is simpler to generate at a desired level of fidelity than discrete waveforms.

Various signal processing methods have been considered for the suppression of impulsive and transient interference signals for enhanced OTHR performance (see for example [6]-[10]). However, to our knowledge, signal detection and cross-radar interference mitigation in a dual-radar OTHR system was not considered until [5]. This paper develops a new cross-radar interference cancellation approach which is similar but more robust compared to the approach in [5]. It is important to note that the OTHR problem addressed here is quite different from the multistatic adaptive radar reception and pulse compression methods developed for other types of radar and synthetic aperture radar (SAR) systems which apply beamforming for spatial processing [11], [12]. In OTHR systems, beamforming is performed primarily for the selection of cross-range bins and thus the spatial selectivity may not be further utilized at subsequent signal processing.

The rest part of this paper is organized as follows. Section II introduces the signal model based on [13] for single-radar operation. This model is extended in Section III to dual-radar operation scenarios, and the effect of cross-radar interference is discussed [5]. Section IV considers the suppression of crossradar interference, and a new cross-radar interference cancellation method is developed. The performance of dual-radar systems as well as the cross-radar interference mitigation is analyzed in Section V. Simulation results using measured clutter data and synthesized target signals are provided in Section VI.

\section{Signal Model}

In this section, we review the signal model based on [13] for the radar applications where the frequency band of interest is solely occupied by a single OTHR system. An OTHR typically employs $\mathrm{FM} / \mathrm{CW}$ signals consisting of a coherent series of chirps to determine the target time-delay (slant range) and Doppler information [14]. Each waveform is an LFM, or chirp, signal of the form

$$
v_{p}(t)= \begin{cases}\exp \left(j \pi B f_{r} t^{2}\right), & 0 \leq t<T_{r} \\ 0, & \text { otherwise }\end{cases}
$$

where $T_{r}$ and $f_{r}=1 / T_{r}$ are, respectively, the waveform repetition interval (WRI) and WRF, and $B$ is the bandwidth of the chirp. The transmitted radar signal, $u(t)$, for the $l$ th revisit consists of a series of $M$ LFM waveforms, i.e.,

$$
u(t)=\alpha_{0} \exp \left(j \omega_{0} t\right) \sum_{m=0}^{M-1} v_{p}\left(t-m T_{r}-t_{l}\right)
$$

where $\alpha_{0}$ is a complex scaler representing the transmitted signal amplitude and phase, $\omega_{0}=2 \pi f$ is the radar operating frequency, and $t_{l}$ is the time of the $l$ th revisit. For $M$ transmitted waveforms, the signal duration, called the coherent integration time (CIT), is $T_{c}=M T_{r}$. In this paper, we only consider one revisit and, therefore, the index of $l$ is omitted thereafter, and $t_{l}=0$ is assumed.

The received signal corresponding to a target $s$ can be expressed as

$$
\begin{aligned}
u_{s}(t)=\tilde{\alpha}_{s} \exp \left[j\left(\omega_{0}+\omega_{s}\right)\left(t-\frac{p_{s}}{c}\right)\right] & \\
& \times \sum_{m=0}^{M-1} v_{p}\left(t-\frac{d_{s}}{c}-m T_{r}\right)+\eta(t)
\end{aligned}
$$

where $\tilde{\alpha}_{s}$ represents the radar return amplitude and phase, $p_{s}$ is the phase path length corresponding to the phase delay, $c$ is the speed of light, $\omega_{s}$ is the Doppler frequency shift, $d_{s}$ is the two-way slant (group) range, and $\eta(t)$ represents additive noise. Note that, while the distances from target $s$ to the radar transmitter and receiver are different, this difference is negligible and only its effect in the phase is considered.

In the process of dechirpping, the received signal is mixed with a delayed version of the transmitted signal, i.e.,

$$
w(t)=u_{s}(t) u^{*}\left(t-T_{0}\right)
$$

where superscript * denotes complex conjugate, and delay $T_{0}$ specifies the minimum delay or start range of the dwell illumination region (DIR). Passing $w(t)$ through a low pass filter (LPF), which eliminates $\omega_{0}$ and $2 \omega_{0}$ components and only retains the baseband component, results in the dechirped signal, $q(t)$, given by

$$
\begin{aligned}
q(t)= & \alpha_{s} \exp \left(j \omega_{s} t\right) \\
& \times \sum_{m=0}^{M-1} \exp \left[-j\left(2 \pi B f_{r}\right)\left(\frac{d_{s}}{c}-T_{0}\right)\left(t-m T_{r}\right)\right] \\
& +\xi(t)
\end{aligned}
$$

where the constant phase and amplitude terms are lumped into $\alpha_{s}$ and where $\xi(t)$ is the low pass filtered noise.

Let $t^{\prime}=t-m T_{r}$. The waveform is sampled at time intervals $t^{\prime}=n T_{s}$, giving the discrete signal $q[m, n]=q_{m}\left(n T_{s}\right)$ as

$$
\begin{aligned}
& q[m, n]=\alpha_{s} \exp \left(j \omega_{s} m T_{r}\right) \\
& \quad \times \exp \left\{j\left[\omega_{s}-2 \pi B f_{r}\left(\frac{d_{s}}{c}-T_{0}\right)\right] n T_{s}\right\}+\xi_{m, n}
\end{aligned}
$$

which is the product of two complex sinusoids in pulse index $m$ and sample index $n$, combined with the additive noise $\xi_{m, n}$. The phase changes over $n$ within one pulse provide slant range information while the phase changes over $m$ from pulse to pulse give Doppler information.

A two-dimensional (2-D) discrete Fourier transform (DFT) of $q[m, n]$ over the two variables $m$ and $n$ gives a complex rangeDoppler surface, $Q\left[\mathrm{~m}^{\prime}, n^{\prime}\right]$. The $N$-point DFT over $n$ within one pulse gives the slant range distribution indexed by $n^{\prime}$, whereas the $M$-point DFT over $m$ across waveforms gives the Doppler frequency distribution indexed by $m^{\prime}$. The slant range bin width of $Q\left[m^{\prime}, n^{\prime}\right]$ is equal to $\Delta d=c /(2 B)$, whereas the Doppler bin width is equal to $\Delta \omega=2 \pi / T_{c}=2 \pi /\left(M T_{r}\right)$. The maximum unambiguous Doppler frequency is $f_{\max }=1 /\left(2 T_{r}\right)$.

To reduce slant range and Doppler sidelobes in $Q\left[m^{\prime}, n^{\prime}\right]$, slant range window $W_{n}$ is applied to $q[m, n]$ over $n$ and Doppler 
window $W_{m}$ is applied to $q[m, n]$ over $m$ before performing the respective DFTs. With the slant range and Doppler windowing, the complex range-Doppler surface can be written as

$$
\begin{aligned}
Q\left[m^{\prime}, n^{\prime}\right]= & \alpha_{s} \operatorname{DFT}_{M}\left[W_{m} \exp \left(j \omega_{s} m T_{r}\right)\right] \\
& \cdot \operatorname{DFT}_{N}\left[W _ { n } \operatorname { e x p } \left\{j \left[\omega_{s}\right.\right.\right. \\
& \left.\left.\left.-2 \pi B f_{r}\left(\frac{d_{s}}{c}-T_{0}\right)\right] n T_{s}\right\}\right]+\xi_{m^{\prime}, n^{\prime}}
\end{aligned}
$$

where $\xi_{m^{\prime}, n^{\prime}}$ is additive noise in the complex range-Doppler domain.

\section{DUAL-RADAR OPERATION}

Now we consider a concurrent operation of two radars which are widely separated. Each radar transmits its own LFM waveform with the same WRF. The two radars are assumed to have carrier frequency alignment and the difference between their ionospheric propagation conditions is not considered. We use the following prototype waveforms of the two radars (the subscripts 1 and 2 represent radars $A$ and $B$, respectively)

$$
\begin{aligned}
& v_{p, 1}(t)=v_{p}(t)= \begin{cases}\exp \left(j \pi B f_{r} t^{2}\right), & 0 \leq t<T_{r} \\
0, & \text { otherwise }\end{cases} \\
& v_{p, 2}(t)=v_{p}^{*}(t)= \begin{cases}\exp \left(-j \pi B f_{r} t^{2}\right), & 0 \leq t<T_{r} \\
0, & \text { otherwise. }\end{cases}
\end{aligned}
$$

Then, the transmitted radar signals become

$$
u_{i}(t)=\alpha_{0, i} \exp \left(j \omega_{0} t\right) \sum_{m=0}^{M-1} v_{p, i}\left(t-m T_{r}\right), \quad i=1,2 .
$$

The received signal from a target $s$ at radar $k(k=1,2)$ corresponding to the signal transmitted from both radar transmitters is expressed as

$$
\begin{array}{r}
u_{s, k}(t)=\sum_{i=1}^{2} \tilde{\alpha}_{s, i, k} \exp \left[j\left(\omega_{0}+\omega_{s, i, k}\right)\left(t-\frac{p_{s, i, k}}{c}\right)\right] \\
\cdot \sum_{m=0}^{M-1} v_{p, i}\left(t-\frac{d_{s, i, k}}{c}-m T_{r}\right)+\eta_{k}(t)
\end{array}
$$

where $\tilde{\alpha}_{s, i, k}, p_{s, i, k}$, and $d_{s, i, k}$ represent the respective magnitude and phase, phase path delay, and two-way slant range for target $s$ with signal transmitted from radar $i$ and received by radar $k$, and $\omega_{s, i, k}$ is the Doppler frequency shift corresponding to transmit radar $i$ and receive radar $k$. In addition, $\eta_{k}(t)$ denotes the additive noise.

Without loss of generality, consider the receive signal at radar A, i.e., $k=1$. Then, the received signal can be expressed in the following three terms

$$
u_{s, 1}(t)=u_{s, 1,1}(t)+u_{s, 2,1}(t)+\eta_{1}(t)
$$

where the first two terms, respectively, represent the contributions of radar $\mathrm{A}$ and radar $\mathrm{B}$. The received signal is then processed using reception modes matched to both radar $\mathrm{A}$ and radar $\mathrm{B}$.

At the reception mode matched to radar $\mathrm{A}$, the following equation is used to dechirp the signal:

$$
\begin{aligned}
& w_{1}(t)=u_{s, 1}(t) u_{1}^{*}\left(t-T_{0}\right)=u_{s, 1,1}(t) u_{1}^{*}\left(t-T_{0}\right) \\
& +u_{s, 2,1}(t) u_{1}^{*}\left(t-T_{0}\right)+\eta_{1}(t) u_{1}^{*}\left(t-T_{0}\right) .
\end{aligned}
$$

The first term of the right-hand side is the auto-correlated term of radar A and is identical to (4) corresponding to the singleradar mode. Therefore, the result of the LPF output, using the new notations defined for the dual-radar scenario, is expressed as

$$
\begin{aligned}
q_{1,1}(t)=\alpha_{s, 1,1} & \exp \left(j \omega_{s, 1,1} t\right) \\
& \cdot \sum_{m=0}^{M-1} \exp \left[-j 2 \pi B f_{r}\left(\frac{d_{s, 1,1}}{c}-T_{0}\right) t^{\prime}\right] .
\end{aligned}
$$

where $t^{\prime}=t-m T_{r}$. The second term is the cross-radar interference term, and its LPF output is expressed as

$$
\begin{aligned}
q_{2,1}(t)= & u_{s, 2,1}(t) u_{1}^{*}\left(t-T_{0}\right) \\
= & \tilde{\alpha}_{s, 2,1} \exp \left[j\left(\omega_{0}+\omega_{s, 2,1}\right)\left(t-\frac{p_{s, 2,1}}{c}\right)\right] \\
& \cdot \sum_{m=0}^{M-1} v_{p, 2}\left(t^{\prime}-\frac{d_{s, 2,1}}{c}\right) \\
& \cdot \alpha_{0,2}^{*} \exp \left(-j \omega_{0} t\right) \sum_{m=0}^{M-1} v_{p, 1}^{*}\left(t^{\prime}-T_{0}\right) \\
= & \tilde{\alpha}_{s, 2,1} \alpha_{0,2}^{*} \exp \left[-j \omega_{0} \frac{\phi_{s, 2,1}}{c}\right. \\
& \left.+j \omega_{s, 2,1}\left(t-\frac{p_{s, 2,1}}{c}\right)\right] \\
& \cdot \sum_{m=0}^{M-1} \exp \left\{-j \pi B f_{r}\left[\left(t^{\prime}-\frac{d_{s, 2,1}}{c}\right)^{2}\right.\right. \\
& \left.\left.+\left(t^{\prime}-T_{0}\right)^{2}\right]\right\} \\
= & \alpha_{s, 2,1} \exp \left(j \omega_{s, 2,1} t\right) \\
& \cdot \sum_{m=0}^{M-1} \exp \left\{-j 2 \pi B f_{r}\left[\left(t^{\prime}\right)^{2}\right.\right. \\
& \left.\left.-\left(\frac{d_{s, 2,1}}{c}+T_{0}\right) t^{\prime}\right]\right\}
\end{aligned}
$$

where $\alpha_{s, 2,1}$ includes the constant phase and amplitudes terms. Similar to the single-radar case, we sample the signal $q_{1,1}(t)$ and $q_{1,2}(t)$ at $t^{\prime}=n T_{s}$, and the results are denoted as $q_{1,1}[m, n]$ and $q_{2,1}[m, n]$, respectively. Then, the 2-D DFT results of $q_{1,1}[m, n]$ and $q_{2,1}[m, n]$ are expressed, respectively, as

$$
\begin{aligned}
Q_{1,1}\left[m^{\prime}, n^{\prime}\right]= & \alpha_{s, 1,1} \operatorname{DFT}_{M}\left[W_{m} \exp \left(j \omega_{s, 1,1} m T_{r}\right)\right] \\
& \cdot \operatorname{DFT}_{N}\left\{W _ { n } \operatorname { e x p } \left[j \left(\omega_{s, 1,1}\right.\right.\right. \\
& \left.\left.\left.-2 \pi B f_{r}\left(\frac{d_{s, 1,1}}{c}-T_{0}\right)\right) n T_{s}\right]\right\}
\end{aligned}
$$




$$
\begin{aligned}
& \text { and } \\
& \begin{aligned}
Q_{2,1}\left[m^{\prime}, n^{\prime}\right]= & \alpha_{s, 2,1} \operatorname{DFT}_{M}\left[W_{m} \exp \left(j \omega_{s, 2,1} m T_{r}\right)\right] \\
& \cdot \operatorname{DFT}_{N}\left\{W _ { n } \operatorname { e x p } \left[-j 2 \pi B f_{r}\left(n T_{s}\right)^{2}\right.\right. \\
& \left.\left.+j\left(\omega_{s, 2,1}+2 \pi B f_{r}\left(\frac{d_{s, 2,1}}{c}+T_{0}\right)\right) n T_{s}\right]\right\} .
\end{aligned}
\end{aligned}
$$

Both components, in addition to the noise, contribute to the complex range-Doppler surface. The signal transmitted from radar $\mathrm{A}$ is the auto-radar response at the radar A reception mode and is localized in both range and Doppler domains. On the other hand, the cross-radar interference only maintains the Doppler information with respect to slow time $T_{r}$. In this case, its rangedomain response is a chirp signal with respect to the fast time $T_{s}$ and the chirp rate is twice that of the transmitted signal. That is, the cross-radar interference keeps the Doppler information whereas the range profile information is lost.

Combining $Q_{1,1}\left[m^{\prime}, n^{\prime}\right]$ and $Q_{2,1}\left[m^{\prime}, n^{\prime}\right]$ as well as noise results in

$$
Q_{1}\left[m^{\prime}, n^{\prime}\right]=Q_{1,1}\left[m^{\prime}, n^{\prime}\right]+Q_{1,1}\left[m^{\prime}, n^{\prime}\right]+\xi_{1}\left[m^{\prime}, n^{\prime}\right] .
$$

In addition to the ordinary operation mode matched to radar A's waveform, radar A can also process the received data using radar B's waveform for dechirping. Due to symmetry, the complex range-Doppler surfaces corresponding to the Radar B reception mode can be readily written as

$$
\tilde{Q}_{1}\left[m^{\prime}, n^{\prime}\right]=\tilde{Q}_{1,1}\left[m^{\prime}, n^{\prime}\right]+\tilde{Q}_{2,1}\left[m^{\prime}, n^{\prime}\right]+\tilde{\xi}_{1}\left[m^{\prime}, n^{\prime}\right]
$$

where

$$
\begin{aligned}
\tilde{Q}_{1,1}\left[m^{\prime}, n^{\prime}\right]= & \alpha_{s, 1,1} \operatorname{DFT}_{M}\left[W_{m} \exp \left(j \omega_{s, 1,1} m T_{r}\right)\right] \\
& \cdot \operatorname{DFT}_{N}\left\{W _ { n } \operatorname { e x p } \left[j 2 \pi B f_{r}\left(n T_{s}\right)^{2}\right.\right. \\
& +j\left(\omega_{s, 1,1}-2 \pi B f_{r}\right. \\
& \left.\left.\left.\times\left(\frac{d_{s, 1,1}}{c}+T_{0}\right)\right) n T_{s}\right]\right\}
\end{aligned}
$$

and

$$
\begin{aligned}
\tilde{Q}_{2,1}\left[m^{\prime}, n^{\prime}\right]= & \alpha_{s, 2,1} \operatorname{DFT}_{M}\left[W_{m} \exp \left(j \omega_{s, 2,1} m T_{r}\right)\right] \\
& \cdot \operatorname{DFT}_{N}\left\{W _ { n } \operatorname { e x p } \left[j \left(\omega_{s, 2,1}\right.\right.\right. \\
& \left.\left.\left.+2 \pi B f_{r}\left(\frac{d_{s, 2,1}}{c}-T_{0}\right)\right) n T_{s}\right]\right\} .
\end{aligned}
$$

In this case, the signal transmitted from radar B is the auto-radar response and is localized in both range and Doppler domains. The signal transmitted from radar A becomes cross-radar interference which is localized only in the Doppler domain.

\section{CROSS-RADAR INTERFERENCE CANCELlation}

As demonstrated in the previous discussion, when both radars are operational, a target will return both radar signals, resulting in a received signal with the desirable signal superimposed a cross-radar interference. In this section, we consider the cancellation of such interference.

\section{A. Technical Challenges}

The interference cancellation process resembles that in the multiuser CDMA communications where dispersive channels are involved [15]-[17]. In the underlying scenario, the chirp signal waveforms act as the spreading codes. However, there are significant differences between the problem at hand and the multiuser CDMA problems. These differences, which prevent the multiuser detection methods to be directly applicable to cross-radar interference cancellation, are summarized as follows.

1) The primary purpose of a CDMA communication system is to deliver information over a multiuser channel. The system is usually designed such that the channels are quasi-stationary, i.e., the channel variation over a certain period is negligible. Therefore, interference cancellation as well as the information detection can be performed within each symbol or over the coherent time of the channels. Usually, processing of a longer period improves the performance, at the expense of higher computational costs. Channel coding is also commonly used to provide additional protection against channel distortion. In the OTHR problems, the information of interest is included in the characteristics of the time-varying channels (clutter and target), whereas the transmit waveform itself does not bear any information. As a result, problems may arise in suppressing cross-radar interference in both single- or multi-waveform period approaches. Processing interference cancellation individually for each waveform may differently alter the phase information and result in wrong Doppler information or Doppler aliasing. On the other hand, joint processing over multiple waveforms requires separate considerations of the clutter and target echo signals because their phase variations due to the Doppler effect differ from each other.

2) In CDMA multiuser detection problems, the aim is to sufficiently reduce the multiuser interference for correct information detection. In the underlying OTHR applications, the signal-to-clutter ratio is very low and a moderate level of interference cancellation is not necessarily sufficient to improve the visibility of the echo signals from moving targets.

3) In CDMA systems, there is redundancy in the signal bandwidth for each user due to spectrum spreading, and the channel order is often limited. Therefore, orthogonal or quasi-orthogonal waveform design for different users is possible. OTHR radar systems, on the other hand, are design to fully utilize the time-frequency resource to obtain the information in the joint range-Doppler domain, and the target may appear in any range cell. As a result, orthogonal waveform design over the entire range cells is impractical. As we discussed in Section I, LFM waveforms are often preferred in OTHR systems. Thus, the reduction of the autoand cross-correlation between the signals for different users and corresponding to different time lags is limited.

The first two issues can be resolved by converting the slow time into the Doppler frequency domain. In doing so, weak signals corresponding to moving targets are separated from strong stationary clutter, which is typically concentrated in low frequencies. In the frequency domain, as we discussed above, the 
time-varying channels corresponding to moving targets become stationary Doppler. In addition, in the Doppler frequency bins where a moving target is positioned, the target signals often have a higher power than that of clutter, making the target detection possible.

For the third issue, we take advantage of the sparseness of the target signals and consider iterative interference cancellation in this paper. The detailed techniques are presented in the next subsection.

\section{B. Interference Cancellation}

For convenience of representation and matrix operation, we rewrite (16) as

$$
\begin{aligned}
Q_{1,1} & {\left[m^{\prime}, n^{\prime}\right] } \\
= & \alpha_{s, 1,1}\left(\mathbf{f}_{m^{\prime}} \mathbf{W}_{M} \mathbf{F}_{M}^{*} \boldsymbol{\epsilon}_{l_{a, 1,1}}\right) \\
& \cdot\left(\mathbf{f}_{n^{\prime}} \mathbf{W}_{N} \cdot \operatorname{diag}\left(\mathbf{v}_{1}^{*}\right) \cdot \mathbf{V}_{1}^{*} \mathbf{e}_{l_{b, 1,1}}\right) \\
= & \alpha_{s, 1,1}\left(\mathbf{f}_{m^{\prime}} \mathbf{W}_{M} \mathbf{F}_{M}^{*} \boldsymbol{\epsilon}_{l_{a, 1,1}}\right) \\
& \cdot\left(\mathbf{f}_{n^{\prime}} \cdot \operatorname{diag}\left(\mathbf{v}_{1}^{*}\right) \cdot \mathbf{W}_{N} \mathbf{V}_{1}^{*} \mathbf{e}_{l_{b, 1,1}}\right)
\end{aligned}
$$

where $\mathbf{F}_{M}\left(\right.$ or $\left.\mathbf{F}_{N}\right)$ denotes the $M \times M($ or $N \times N)$ Fourier transform matrix, $\mathbf{f}_{m^{\prime}}$ (or $\mathbf{f}_{n^{\prime}}$ ) denotes the $m^{\prime}$ th (or $n^{\prime}$ th) row of $\mathbf{F}_{M}\left(\right.$ or $\left.\mathbf{F}_{N}\right), \mathbf{W}_{M}\left(\right.$ or $\left.\mathbf{W}_{N}\right)$ is an $M \times M($ or $N \times N)$ diagonal matrix with proper window coefficients as its diagonal elements, $\operatorname{diag}\left(\mathbf{v}_{1}\right)$ is a diagonal matrix with

$$
\mathbf{v}_{1}=\left[v_{p, i}[0], v_{p, i}[1], \cdots, v_{p, i}[N-1]\right]
$$

as its diagonal elements, where $v_{p, i}[n]=v_{p, i}\left(n T_{s}\right)$. In addition

$$
\mathbf{V}_{i}=\left[\begin{array}{ccccc}
v_{p, i}[0] & v_{p, i}[1] & \cdots & v_{p, i}[N-2] & v_{p, i}[N-1] \\
v_{p, i}[N-1] & v_{p, i}[0] & \cdots & v_{p, i}[N-3] & v_{p, i}[N-2] \\
\cdots & \cdots & \cdots & \cdots & \cdots \\
v_{p, i}[1] & v_{p, i}[2] & \cdots & v_{p, i}[N-1] & v_{p, i}[0]
\end{array}\right]
$$$$
i=1,2 \text {, }
$$

and $\epsilon_{l}$ and $\mathbf{e}_{l}$ are, respectively, $M \times 1$ and $2 N \times 1$ vectors with all zeros except a unit value at the $l$ th element. For a target whose Doppler radian frequency with respect to radar $\mathrm{A}$ is $\omega_{s, 1,1}$ and its range is $d_{s, 1,1}$, we have

$$
l_{a, 1,1}=\frac{M}{2 \pi} \omega_{s, 1,1} T_{r}+1
$$

and

$$
l_{b, 1,1}=N f_{r}\left(\frac{d_{s, 1,1}}{c}-T_{0}\right)-\frac{N \omega_{s, 1,1}}{2 \pi B}+1 .
$$

Typically, the contribution of $\omega_{s, 1,1}$ to the short-time frequency shift is negligible, and $l_{b, 1,1}$ is primarily determined by the range $d_{s, 1,1}$ relative to the reference time $T_{0}$.

Stacking $Q_{1,1}\left[m^{\prime}, n^{\prime}\right]$ for the $N$ range cells $n^{\prime}=0, \ldots, N-$ 1 , we obtain

$$
\begin{aligned}
\mathbf{q}_{1,1}\left[m^{\prime}\right]= & {\left[\begin{array}{c}
Q_{1,1}\left[m^{\prime}, 0\right]+Q_{2,1}\left[m^{\prime}, 0\right] \\
\ldots \\
Q_{1,1}\left[m^{\prime}, N-1\right]+Q_{2,1}\left[m^{\prime}, N-1\right]
\end{array}\right] } \\
= & \alpha_{s, 1,1}\left(\mathbf{f}_{m^{\prime}} \mathbf{W}_{M} \mathbf{F}_{M}^{*} \boldsymbol{\epsilon}_{l_{a, 1,1}}\right) \\
& \cdot\left(\mathbf{F}_{N} \cdot \operatorname{diag}\left(\mathbf{v}_{1}^{*}\right) \cdot \mathbf{W}_{N} \mathbf{V}_{1} \mathbf{e}_{l_{b, 1,1}}\right) .
\end{aligned}
$$

Note that, in practice, the received signal is often oversampled (i.e., $N>B T_{r}$ ) to achieve an enhanced range resolution. In this case, the $N$ frequency bins of $\mathbf{F}_{N}$ are chosen to span the signal bandwidth $[-B / 2, B / 2]$, rather than the entire viewable bandwidth $\left[-1 / 2 T_{s}, 1 / 2 T_{s}\right]$. Thus, we can rewrite the above expression as

$$
\begin{array}{r}
\mathbf{q}_{1,1}\left[m^{\prime}\right]=\alpha_{s, 1,1}\left(\mathbf{f}_{m^{\prime}} \mathbf{W}_{M} \mathbf{F}_{M}^{*} \boldsymbol{\epsilon}_{l_{a, 1,1}}\right) \\
\cdot\left(\mathbf{V}_{1}^{H} \mathbf{W}_{N} \mathbf{V}_{1} \mathbf{e}_{l_{b, 1,1}}\right) .
\end{array}
$$

Note that, while we used the same notation $\mathbf{q}_{1,1}\left[\mathrm{~m}^{\prime}\right]$ in both (27) and (28), the results in these two equations differ in their phase. Nevertheless, for simplicity, no distinctions are made between the two variables, as only their respective amplitudes affect target detection.

In general, $l_{a, 1,1}$ and $l_{b, 1,1}$ do not necessarily take integer values, resulting in smeared representations over multiple neighboring range bins. Furthermore, in practice, clutter arises due to a collection of reflection and scattering from earth or ocean surfaces. When multiple targets exist, the reflected signal may arrive through a single or multiple paths. Therefore, we generalize the two vectors $\boldsymbol{\epsilon}_{l_{a, 1,1}}$ and $\mathbf{e}_{l_{b, 1,1}}$ into arbitrary $M \times 1$ vector $\mathbf{h}_{a, 1,1}$ and $N \times 1$ vector $\mathbf{h}_{b, 1,1}$, respectively, to represent the collective contributions from targets and clutters. Similarly, we denote $\mathbf{h}_{a, 2,1}$ and $\mathbf{h}_{b, 2,1}$ as the contribution corresponding to signal transmitted from radar B. Notice that the clutter energy is highly localized in very low Doppler frequencies and cannot be resolved in the range domain, whereas the target energy typically positions away from the zero Doppler frequencies and is localized in range.

Rewriting (28) by using $\mathbf{h}_{a, 1,1}$ and $\mathbf{h}_{b, 1,1}$, and deriving $\mathbf{q}_{2,1}\left[m^{\prime}\right], \tilde{\mathbf{q}}_{1,2}\left[m^{\prime}\right]$, and $\tilde{\mathbf{q}}_{2,2}\left[m^{\prime}\right]$ corresponding to (17), (20), and (21), respectively, we have

$$
\begin{aligned}
\mathbf{q}_{1,1}\left[m^{\prime}\right]= & \alpha_{s, 1,1}\left(\mathbf{f}_{m} \mathbf{W}_{M} \mathbf{F}_{M}^{*} \mathbf{h}_{a, 1,1}\right) \\
& \cdot\left(\mathbf{V}_{1}^{H} \mathbf{W}_{N} \mathbf{V}_{1} \mathbf{h}_{b, 1,1}\right) \\
\mathbf{q}_{2,1}\left[m^{\prime}\right]= & \alpha_{s, 2,1}\left(\mathbf{f}_{m} \mathbf{W}_{M} \mathbf{F}_{M}^{*} \mathbf{h}_{a, 2,1}\right) \\
& \cdot\left(\mathbf{V}_{1}^{H} \mathbf{W}_{N} \mathbf{V}_{2} \mathbf{h}_{b, 2,1}\right) \\
\tilde{\mathbf{q}}_{1,1}\left[m^{\prime}\right]= & \alpha_{s, 1,1}\left(\mathbf{f}_{m} \mathbf{W}_{M} \mathbf{F}_{M}^{*} \mathbf{h}_{a, 1,1}\right) \\
& \cdot\left(\mathbf{V}_{2}^{H} \mathbf{W}_{N} \mathbf{V}_{1} \mathbf{h}_{b, 1,1}\right) \\
\tilde{\mathbf{q}}_{2,1}\left[m^{\prime}\right]= & \alpha_{s, 2,1}\left(\mathbf{f}_{m} \mathbf{W}_{M} \mathbf{F}_{M}^{*} \mathbf{e}_{l_{a, 2,1}}\right) \\
& \cdot\left(\mathbf{V}_{2}^{H} \mathbf{W}_{N} \mathbf{V}_{2} \mathbf{h}_{b, 2,1}\right) .
\end{aligned}
$$

Therefore, at the $m^{\prime}$ th Doppler frequency bin, these vectors can be combined to form a $2 N \times 1$ vector, expressed as

$$
\begin{aligned}
& \mathbf{q}_{1}\left[m^{\prime}\right]= {\left[\begin{array}{l}
\mathbf{q}_{1,1}\left[m^{\prime}\right]+\mathbf{q}_{2,1}\left[m^{\prime}\right] \\
\tilde{\mathbf{q}}_{1,1}\left[m^{\prime}\right]+\tilde{\mathbf{q}}_{2,1}\left[m^{\prime}\right]
\end{array}\right] } \\
&= {\left[\begin{array}{l}
\mathbf{V}_{1}^{H} \\
\mathbf{V}_{2}^{H}
\end{array}\right] \mathbf{W}_{N}\left[\mathbf{V}_{1} \mathbf{V}_{2}\right] } \\
& \times\left[\begin{array}{l}
\mathbf{h}_{b, 1,1} \alpha_{s, 1,1} \mathbf{f}_{m} \mathbf{W}_{M} \mathbf{F}^{\star}{ }^{\mathbf{h}_{a, 1,1}} \\
\mathbf{h}_{b, 2,1} \alpha_{s, 2,1} \mathbf{f}_{m} \mathbf{W}_{M} \mathbf{F}^{\star} \mathbf{h}_{a, 2,1}
\end{array}\right] \\
& \stackrel{\text { def }}{=}\left[\begin{array}{c}
\mathbf{V}_{1}^{H} \\
\mathbf{V}_{2}^{H}
\end{array}\right] \mathbf{W}_{N}\left[\mathbf{V}_{1} \mathbf{V}_{2}\right]\left[\begin{array}{l}
\tilde{\mathbf{h}}_{b, 1,1}\left[m^{\prime}\right] \\
\tilde{\mathbf{h}}_{b, 2,1}\left[m^{\prime}\right]
\end{array}\right] .
\end{aligned}
$$


The upper $N$ elements of $\mathbf{q}_{1}\left[\mathrm{~m}^{\prime}\right]$ represents the auto-radar responses in the range-Doppler domain, whereas the lower $N$ elements denotes the cross-radar interference. In the above equation, $\alpha_{s, 1,1} \mathbf{f}_{m} \mathbf{W}_{M} \mathbf{F}_{M}^{*} \mathbf{h}_{a, 1,1}$ and $\alpha_{s, 2,1} \mathbf{f}_{m} \mathbf{W}_{M} \mathbf{F}_{M}^{*} \mathbf{h}_{a, 2,1}$ are two complex scalars representing the overall strength in Doppler frequency bin $m^{\prime}$. They are multiplied by vectors $\mathbf{h}_{b, l, 1}$ and $\mathbf{h}_{b, 2,1}$, respectively, to form a vector $\tilde{\mathbf{h}}_{b, 1}=\left[\tilde{\mathbf{h}}_{b, l, 1}^{T}\left[m^{\prime}\right], \tilde{\mathbf{h}}_{b, 2,1}^{T}\left[m^{\prime}\right]\right]^{T}$ of $2 N$ unknown (dependent) elements depicting the contribution from targets and scatterers in the $N$ range cells, corresponding to the signals transmitted from radar A (auto-radar reception mode) and radar B (cross-radar reception mode). Our goal in this section is to eliminate the cross-radar interference without compromising the auto-radar responses. The return signals due to radar A and radar B cannot be directly separated because matrix $\mathbf{V}=\left[\mathbf{V}_{1}, \mathbf{V}_{2}\right]$ involved in the above expression is a wide matrix. In other words, although we have $2 N$ observations $\left(\mathbf{q}_{1}\left[\mathrm{~m}^{\prime}\right]\right.$ obtained using both radar $\mathrm{A}$ and radar $\mathrm{B}$ reception modes) and the aforementioned $2 N$ unknowns, the problem cannot be directly solved as the observation vector is rank deficient.

Below, we employ iterative interference cancellation method to take advantage of the fact that the signals of interest which arise from moving targets are typically sparse in range. We first compute the $2 N \times 2 N$ matrix

$$
\tilde{\mathbf{Q}}_{1}=\mathbf{V}^{H} \mathbf{W}_{N} \mathbf{V} \mathbf{I}_{2 N}=\mathbf{V}^{H} \mathbf{W}_{N} \mathbf{V}
$$

i.e., a collection of the response of $\mathbf{q}_{1}$ corresponding to all $2 N$ possible impulse values of $\tilde{\mathbf{h}}_{b, 1,1}$ or $\tilde{\mathbf{h}}_{b, 2,1}$ being $\mathbf{e}_{i}, i=1, \cdots, 2 N$, where $\mathbf{I}_{2 N}$ is the $2 N \times 2 N$ identity matrix. Note that the above result is independent of $m^{\prime}$. Also, we compute $\mathbf{q}_{1}\left[m^{\prime}\right]$ based on (33) for $m^{\prime}=0, \cdots, M-1$.

For each frequency bin index $m^{\prime}$, the following steps are performed in each iteration. The concept behind the iterative cross-radar interference cancellation is similar to the CLEAN techniques [18]-[20].

1) Find the maximum value $q_{1, \max }^{(l)}\left[m^{\prime}\right]=\left[\mathbf{q}_{1}^{(l)}\left[m^{\prime}\right]\right]_{n^{\prime *}}$, where $n^{\prime *}$ is the position of the maximum value. We use ${ }^{(l)}$ to identify the $l$ th iteration and define $\mathbf{q}_{1}^{(1)}\left[\mathrm{m}^{\prime}\right] \stackrel{\text { def }}{=} \mathbf{q}_{1}\left[\mathrm{~m}^{\prime}\right]$. Thus, it can be considered that there is a point source at the $n^{*}$ th range cell.

2) Compute the cross-radar interference-free Doppler-range response corresponding to the point source at the $n^{\prime *}$ th range cell as

$$
\hat{\mathbf{q}}_{1}^{(l)}\left[m^{\prime}\right]=\left\{\begin{array}{r}
\tilde{\mathbf{q}}_{1}^{(A)}\left[n^{\prime *}\right] \cdot \tilde{\mathbf{q}}_{1}^{H}\left[n^{\prime *}\right] \cdot \mathbf{q}_{1}^{(l)}\left[m^{\prime}\right] /\left|\tilde{\mathbf{q}}_{1}\left[n^{\prime *}\right]\right|^{2} \\
0 \leq n^{\prime *} \leq N-1 \\
\tilde{\mathbf{q}}_{1}^{(B)}\left[n^{\prime *}\right] \cdot \tilde{\mathbf{q}}_{1}^{H}\left[n^{\prime *}\right] \cdot \mathbf{q}_{1}^{(1)}\left[m^{\prime}\right] /\left|\tilde{\mathbf{q}}_{1}\left[n^{\prime *}\right]\right|^{2} \\
N \leq n^{\prime *} \leq 2 N-1
\end{array}\right.
$$

where

$$
\tilde{\mathbf{q}}_{1}\left[n^{\prime *}\right]=\tilde{\mathbf{Q}}_{1} \mathbf{e}_{n^{\prime *}}=\mathbf{V}^{H} \mathbf{W}_{N} \mathbf{V} \mathbf{e}_{n^{\prime *}}
$$

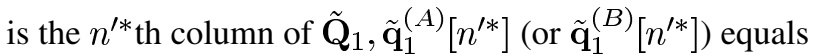
to $\mathbf{q}_{1}\left[n^{\prime *}\right]$ with the second $N$ elements (or the first $N$ element) set to zero.
3) Remove the cross-radar interference-contaminated Doppler-range response corresponding to the point source at the $n^{\prime *}$ th range cell from the residual response by updating the residual response as

$$
\mathbf{q}_{1}^{(l+1)}\left[m^{\prime}\right]=\mathbf{q}_{1}^{(l)}\left[m^{\prime}\right]-\tilde{\mathbf{q}}_{1}\left[n^{\prime *}\right] \tilde{\mathbf{q}}_{1}^{H}\left[n^{\prime *}\right] \mathbf{q}_{1}^{(l)}\left[m^{\prime}\right] /\left|\tilde{\mathbf{q}}_{1}\left[n^{\prime *}\right]\right|^{2} .
$$

The number of iterations can be either prefixed or adaptively determined by the level of the peak residual energy. The signal waveform after $L$ iterations of cross-radar interference mitigation is obtained as $\mathbf{q}_{1}^{(L+1)}\left[m^{\prime}\right]+\sum_{l=1}^{L} \hat{\mathbf{q}}_{1}^{(l)}\left[m^{\prime}\right]$.

\section{Performance Analysis}

To analyze the performance of the dual-radar system as well as the effectiveness of cross-radar interference reduction, we consider a frequency bin $m^{\prime}$ at which a point target is positioned corresponding to the waveform of radar A and thus generate cross-radar interference when it is received to match the waveform of radar B. Due to the symmetry, only the performance at radar $\mathrm{A}$ is considered. We assume that a target is located at the $k$ th range cell and its return signal due to radar A's transmitted waveforms falls at the $m^{\prime}$ th Doppler frequency bin.

We start with (33) and express the channel model as

$$
\tilde{\mathbf{h}}_{b, 1}=\alpha \mathbf{e}_{k}+\mathbf{z}_{1}
$$

where $\alpha \mathbf{e}_{k}$ represents a localized target in the $k$ th range cell, and $\mathbf{z}_{1}=\left[\mathbf{z}_{1,1}^{T}, \mathbf{z}_{2,1}^{T}\right]^{T}$ is the external noise vector. Note that index $m^{\prime}$ is omitted in this section for the simplicity of expression, because only frequency bin $m^{\prime}$ is considered in this section. The internal thermal noise is typically of much less impact compared to clutter and its contribution is neglected in the analysis. We are most interested in the Doppler frequencies that are not very low, thus it is justified to assume that the elements of $\mathbf{z}_{1}$ are independent and identically distributed (i.i.d.) complex Gaussian random variables with zero mean and joint variance matrix $\sigma_{z}^{2} \mathbf{I}_{2 N}$. Note that $\sigma_{z}^{2}$ depends on Doppler bin index $m^{\prime}$.

Without loss of generality, we assume that $\mathbf{v}_{i}$ is normalized to have a unit norm, i.e., $\left|\mathbf{v}_{i}\right|^{2}=\mathbf{v}_{i}^{H} \mathbf{v}_{i}=1$ for $i=1,2$. As each elements of $\mathbf{v}_{i}$ is of constant magnitude, we have $\left[\mathbf{v}_{i}\right]_{k}^{*}\left[\mathbf{v}_{i}\right]_{k}=$ $1 / N$. For the convenience of understanding to the performance, some numerical results are provided for the radar system setting illustrated in Table I. From these parameters, the number of sweeps is $M=T_{c} f_{r}=256$ and the number of range cells is $N=f_{s} / f_{r}=400$.

\section{A. Single Radar Operation}

As the baseline, we first consider the situation where only a single radar (i.e., radar A) is operated. In this case, the received signal is expressed as

$$
\tilde{\mathbf{h}}_{b, 1}^{(A)}=\alpha \mathbf{e}_{k}^{(A)}+\mathbf{z}_{1,1}
$$


where $\tilde{\mathbf{h}}_{b, 1}^{(A)}$ denotes an $N \times 1$ vector consisting of the first $N$ elements of $\tilde{\mathbf{h}}_{b, 1}$ and, similarly, $\mathbf{e}_{k}^{(A)}$ denotes an $N \times 1$ vector consisting of the first $N$ elements of $\mathbf{e}_{k}$. In this case, we obtain

$$
\begin{aligned}
\mathbf{q}_{1} & =\mathbf{q}_{1,1}=\mathbf{V}_{1}^{H} \mathbf{W}_{N} \mathbf{V}_{1} \tilde{\mathbf{h}}_{b, 1}^{(A)} \\
& =\mathbf{V}_{1}^{H} \mathbf{W}_{N} \mathbf{V}_{1}\left[\alpha \mathbf{e}_{k}^{(A)}+\mathbf{z}_{1,1}\right] .
\end{aligned}
$$

For range cells that are separated from the $k$ th range cell by more than the mainlobe width, the effect of target signal is small, particularly when a proper window is used. Thus, we only consider the signal component at the $k$ th range cell which is expressed as

$$
\left[\mathbf{q}_{1}^{(s)}\right]_{k}=\alpha\left[\mathbf{e}_{k}^{(A)}\right]^{T} \mathbf{V}_{1}^{H} \mathbf{W}_{N} \mathbf{V}_{1} \mathbf{e}_{k}^{(A)}=\alpha A_{W}
$$

where $A_{W}=(1 / N) \sum_{i=1}^{N} \operatorname{diag}\left[\mathbf{W}_{N}\right]$ is the average value of the window coefficients of $\mathbf{W}_{N}$. The noise component in (40) is

$$
\mathbf{q}_{1}^{(z)}=\mathbf{V}_{1}^{H} \mathbf{W}_{N} \mathbf{V}_{1} \mathbf{z}_{1,1}
$$

which has a zero mean and covariance matrix

$$
\begin{aligned}
\operatorname{var}\left[\mathbf{q}_{1}^{(z)}\right] & =E\left[\mathbf{V}_{1}^{H} \mathbf{W}_{N} \mathbf{V}_{1} \mathbf{z}_{1,1} \mathbf{z}_{1,1}^{H} \mathbf{V}_{1}^{H} \mathbf{W}_{N} \mathbf{V}_{1}\right] \\
& =\sigma_{z}^{2}\left[\mathbf{V}_{1}^{H} \mathbf{W}_{N} \mathbf{V}_{1} \mathbf{V}_{1}^{H} \mathbf{W}_{N} \mathbf{V}_{1}\right]
\end{aligned}
$$

where $E[\cdot]$ denotes statistical expectation. The $n^{\prime}$ th diagonal elements of the above covariance matrix, denoted as $\left[\operatorname{var}\left[\mathbf{q}_{1}^{(z)}\right]\right]_{n^{\prime}, n^{\prime}}=\sigma_{z}^{2}\left[\mathbf{V}_{1}^{H} \mathbf{W}_{N} \mathbf{V}_{1} \mathbf{V}_{1}^{H} \mathbf{W}_{N} \mathbf{V}_{1}\right]_{n^{\prime}, n^{\prime}}=\gamma \sigma_{z}^{2}$, represents the noise auto-variance at each range cell whose values depends on the sampling rate, signal bandwidth, and the selected window.

Therefore, the signal-to-noise ratio (SNR), evaluated at the range-Doppler result $\mathbf{q}_{1}$, is obtained as

$$
\mathrm{SNR}_{\text {single }}=\frac{|\alpha|^{2} A_{W}^{2}}{\gamma \sigma_{z}^{2}} .
$$

For the parameters listed in Table I, the auto-variance $\gamma \sigma_{z}^{2}$ is about $2.5 \sigma_{z}^{2}$ (without window) and $0.94 \sigma_{z}^{2}$ (with Hanning window), yielding SNR values of $\left(\mathrm{SNR}_{0}-4\right) \mathrm{dB}$ or $\left(\mathrm{SNR}_{0}-5.7\right) \mathrm{dB}$, respectively, without and with the window, where $\mathrm{SNR}_{0}=10 \log _{10}\left(|\alpha|^{2} / \sigma_{z}^{2}\right) \mathrm{dB}$.

\section{B. Dual-Radar Operation}

Now we consider the situation where two radars concurrently transmit signals. For simplicity and without loss of generality, we assume that only the signal transmitted from radar A generate target return at the $m^{\prime}$ th Doppler frequency bin. Due to the linearity of radar system response, it is straightforward to extend the results to multiple targets or the situation where the return signals arisen from both radar A and radar B have the same Doppler frequency.

In the assumed situation, we have

$$
\mathbf{q}_{1}=\mathbf{V}^{H} \mathbf{W}_{N} \mathbf{V} \tilde{\mathbf{h}}_{b, 1}=\mathbf{V}^{H} \mathbf{W}_{N} \mathbf{V}\left[\alpha \mathbf{e}_{k}+\mathbf{z}_{1}\right] .
$$

TABLE I

RADAR SYSTEM PARAMETERS

\begin{tabular}{|c|rl|}
\hline Parameter & Value & (Unit) \\
\hline Carrier frequency $(f)$ & 13.957 & $\mathrm{MHz}$ \\
Signal bandwidth $(B)$ & 8 & $\mathrm{KHz}$ \\
Waveform repetition frequency $\left(f_{r}\right)$ & 50 & $\mathrm{~Hz}$ \\
Sampling frequency $\left(f_{s}\right)$ & 20 & $\mathrm{KHz}$ \\
Coherent integration time $\left(T_{c}\right)$ & 5.12 & $\mathrm{sec}$ \\
\hline
\end{tabular}

It is obvious that the signal component $\left[\mathbf{q}_{1}^{(s)}\right]_{l}$ remains the same as (41). The noise vector

$$
\begin{aligned}
\mathbf{q}_{1}^{(z)} & =\mathbf{V}^{H} \mathbf{W}_{N} \mathbf{V} \mathbf{z}_{1} \\
& =\mathbf{V}^{H} \mathbf{W}_{N} \mathbf{V}_{1} \mathbf{z}_{1,1}+\mathbf{V}^{H} \mathbf{W}_{N} \mathbf{V}_{2} \mathbf{z}_{2,1}
\end{aligned}
$$

has a zero mean, and its covariance matrix is obtained as

$$
\begin{aligned}
\operatorname{var}\left[\mathbf{q}_{1}^{(z)}\right]= & E\left[\mathbf{V}^{H} \mathbf{W}_{N} \mathbf{V} \mathbf{z}_{1} \mathbf{z}_{1}^{H} \mathbf{V}^{H} \mathbf{W}_{N} \mathbf{V}\right] \\
& +\sigma_{z}^{2}\left[\mathbf{V}^{H} \mathbf{W}_{N}\left(\mathbf{V}_{1} \mathbf{V}_{1}^{H}+\mathbf{V}_{2} \mathbf{V}_{2}^{H}\right) \mathbf{W}_{N} \mathbf{V}\right] \\
= & 2 \sigma_{z}^{2}\left[\mathbf{V}^{H} \mathbf{W}_{N} \mathbf{V}_{1} \mathbf{V}_{1}^{H} \mathbf{W}_{N} \mathbf{V}\right] .
\end{aligned}
$$

That is, a $3 \mathrm{~dB}$ clutter enhancement is introduced because of the dual radar operations. As a result, the SNR becomes

$$
\mathrm{SNR}_{\text {dual }}=\frac{|\alpha|^{2} A_{W}^{2}}{2 \gamma \sigma_{z}^{2}} .
$$

In addition, the concurrent use of two radars causes crossradar interference. When radar A processes the received signal with radar B mode, the cross-radar interference becomes

$$
\begin{aligned}
\mathbf{q}_{1}^{(i)} & =\mathbf{L}^{(B)} \mathbf{q}_{1}^{(s)}=\alpha \mathbf{V}_{2}^{H} \mathbf{W}_{N} \mathbf{V e}_{k} \\
& =\alpha \mathbf{V}_{2}^{H} \mathbf{W}_{N} \mathbf{V}_{1} \mathbf{e}_{k}^{(A)}
\end{aligned}
$$

where $\mathbf{L}^{(B)}=\left[\mathbf{O}_{N}, \mathbf{I}_{N}\right]$ with $\mathbf{O}_{N}$ denoting the $N \times N$ zero matrix. As we discussed earlier, the cross-radar interference spans the entire range cells, and its power averaged over the $N$ range cells is obtained as

$$
\begin{aligned}
\frac{1}{N}\left[\mathbf{q}_{1}^{(i)}\right]^{H} \mathbf{q}_{1}^{(i)} & =\frac{|\alpha|^{2}}{N} \mathbf{e}_{k}^{(A)} \mathbf{V}_{1}^{H} \mathbf{W}_{N} \mathbf{V}_{2} \mathbf{V}_{2}^{H} \mathbf{W}_{N} \mathbf{V}_{1} \mathbf{e}_{k}^{(A)} \\
& =\frac{\gamma|\alpha|^{2}}{N} .
\end{aligned}
$$

Therefore, the resulting average signal-to-interference ratio (SIR) is

$$
\operatorname{SIR}_{\text {dual }}=\frac{N A_{W}^{2}}{\gamma}
$$

which, for the parameters given in Table $\mathrm{I}$, is about $22 \mathrm{~dB}$ without a window or $20.3 \mathrm{~dB}$ with the Hanning window.

\section{Performance of Interference Cancellation}

Consider one iteration of the interference cancellation process. We obtain the final result as the sum of two components, 
$\hat{\mathbf{q}}_{1}^{(1)}$ and $\mathbf{q}_{1}^{(2)}$, expressed as

$$
\begin{aligned}
\hat{\mathbf{q}}_{1}^{(1)}+\mathbf{q}_{1}^{(2)}= & \mathbf{q}_{1}-\tilde{\mathbf{q}}_{1}[k] \cdot \tilde{\mathbf{q}}_{1}^{H}[k] \cdot \mathbf{q}_{1} /\left|\tilde{\mathbf{q}}_{1}[k]\right|^{2} \\
& +\tilde{\mathbf{q}}_{1}^{(A)}[k] \cdot \tilde{\mathbf{q}}_{1}^{H}[k] \cdot \mathbf{q}_{1} /\left|\tilde{\mathbf{q}}_{1}[k]\right|^{2} \\
= & \mathbf{q}_{1}-\tilde{\mathbf{q}}_{1}^{(B)}[k] \cdot \tilde{\mathbf{q}}_{1}^{H}[k] \cdot \mathbf{q}_{1} /\left|\tilde{\mathbf{q}}_{1}[k]\right|^{2} .
\end{aligned}
$$

Evidently, the interference cancellation process does not change the result corresponding to the radar A mode, thus the SNR in the auto-radar reception mode remains unchanged, i.e.,

$$
\mathrm{SNR}_{\text {cancel }}=\frac{|\alpha|^{2} A_{W}^{2}}{2 \gamma \sigma_{z}^{2}} \text {. }
$$

On the other hand, we can show that the cross-radar interference due to the target return is totally eliminated. At the cross-radar reception mode, we have

$$
\begin{aligned}
\mathbf{L}^{(B)} & {\left[\hat{\mathbf{q}}_{1}^{(1)}+\mathbf{q}_{1}^{(2)}\right]^{(s)} } \\
= & \mathbf{L}^{(B)} \mathbf{q}_{1}^{(s)}-\mathbf{L}^{(B)} \tilde{\mathbf{q}}_{1}^{(B)}[k] \cdot \tilde{\mathbf{q}}_{1}^{H}[k] \\
& \cdot \alpha \mathbf{V}^{H} \mathbf{W}_{N} \mathbf{V} \mathbf{e}_{k} /\left|\tilde{\mathbf{q}}_{1}[k]\right|^{2} \\
= & \alpha \mathbf{V}_{2}^{H} \mathbf{W}_{N} \mathbf{V e} \mathbf{e}_{k} \\
& -\frac{\alpha \mathbf{V}_{2}^{H} \mathbf{W}_{N} \mathbf{V} \mathbf{e}_{k} \mathbf{e}_{k}^{H} \mathbf{V}^{H} \mathbf{W}_{N} \mathbf{V} \mathbf{V}^{H} \mathbf{W}_{N} \mathbf{V} \mathbf{e}_{k}}{\mathbf{e}_{k}^{H} \mathbf{V}^{H} \mathbf{W}_{N} \mathbf{V} \mathbf{V}^{H} \mathbf{W}_{N} \mathbf{V e} \mathbf{e}_{k}} \\
= & 0 .
\end{aligned}
$$

The residual undesired signal components after cross-radar interference cancellation are made up of the original noise and the residual error due to the erroneous estimation of $\mathbf{q}_{1}$ at the $k$ th range cell. They are given by

$$
\begin{aligned}
\mathbf{q}_{1, \text { cancel }}^{(z)} & \\
= & \mathbf{L}^{(B)}\left[\hat{\mathbf{q}}_{1}^{(1)}+\mathbf{q}_{1}^{(2)}\right]^{(z)} \\
= & \mathbf{L}^{(B)} \mathbf{q}_{1}^{(z)}-\mathbf{L}^{(B)} \tilde{\mathbf{q}}_{1}^{(B)}[k] \cdot \tilde{\mathbf{q}}_{1}^{H}[k] \\
& \cdot \mathbf{V}^{H} \mathbf{W}_{N} \mathbf{V} \mathbf{z}_{1} /\left|\tilde{\mathbf{q}}_{1}[k]\right|^{2} \\
= & \mathbf{L}^{(B)} \mathbf{V}^{H} \mathbf{W}_{N} \mathbf{V} \mathbf{z}_{1} \\
& -\frac{\mathbf{V}_{2}^{H} \mathbf{W}_{N} \mathbf{V e}_{k} \mathbf{e}_{k}^{H} \mathbf{V}^{H} \mathbf{W}_{N} \mathbf{V} \mathbf{V}^{H} \mathbf{W}_{N} \mathbf{V} \mathbf{z}_{1}}{\mathbf{e}_{k}^{H} \mathbf{V}^{H} \mathbf{W}_{N} \mathbf{V} \mathbf{V}^{H} \mathbf{W}_{N} \mathbf{V} \mathbf{e}_{k}} \\
= & \mathbf{L}^{(B)} \mathbf{V}^{H} \mathbf{W}_{N} \mathbf{V} \mathbf{z}_{1} \\
& -\frac{1}{2 \gamma} \mathbf{V}_{2}^{H} \mathbf{W}_{N} \mathbf{V} \mathbf{e}_{k} \mathbf{e}_{k}^{H}\left[\mathbf{V}^{H} \mathbf{W}_{N} \mathbf{V}\right]^{2} \mathbf{z}_{1}
\end{aligned}
$$

where $\mathbf{A}^{2}=\mathbf{A} \mathbf{A}$. Consider the fact that the noise variance before the interference cancellation is the same for both radar reception modes and over all range cells. The operation in the second term of the above equation is a projection of random noise components to a structured vector of unit norm. The power of this term (i.e., the residual error) is much smaller than that of the first term at the right-hand side (i.e., the original noise). That is, the additional noise introduced by the cross-radar interference cancellation process is insignificant. For example, for the parameters provided in Table I, the average power difference between these two terms is $17.6 \mathrm{~dB}$, and the difference becomes about $19 \mathrm{~dB}$ when a Hanning window is applied.
In practice, there is no need to distinguish the two terms in the above equation. We can express the output signal-to-interference-plus-noise ratio (SINR) at the cross-radar reception mode as

$$
\operatorname{SINR}_{\text {cancel }}=\frac{|\alpha|^{2} A_{W}^{2}}{\operatorname{var}\left[\mathbf{q}_{1, \text { cancel }}^{(z)}\right]} \approx \frac{|\alpha|^{2} A_{W}^{2}}{2 \gamma \sigma_{z}^{2}} .
$$

We point out that the actual level of residual error may be higher due to several reasons, primarily the incomplete cancellation of cross-range interference when the target return energy at the auto-radar reception mode is smeared or distributed in the rangeDoppler domain.

\section{Simulation Results}

We have conducted simulations using measured clutter data. A synthetic test target has been injected into the received time series to provide a reference for the assessment of the performance in target SNR depending on processing options. Key parameters of the radar system are shown in Table I, where the data consists of a 256-sweep duration. The Doppler frequency corresponding to the Tx A - target - Rx A path is $10 \mathrm{~Hz}$, and that corresponding to the Tx B - target - Rx A path is $14 \mathrm{~Hz}$. The propagation delay of both paths relative to the reference time is $8 \mathrm{~ms}$. For the convenience of visualizing the effect of cross-radar interference and assessing the effectiveness of interference cancellation, the strength of the synthesized target is set to be relatively high. The SNR and SIR results are assessed for the Tx A - target - Rx A path at the $10 \mathrm{~Hz}$ Doppler frequency bin. Background noise power is obtained when no target returns are injected.

When the two radar systems concurrently transmit, the time-frequency signature is the superposition of the results of the two radar systems, as depicted in Fig. 2(a) using short-time Fourier transform (STFT), where appropriate windows are applied. Fig. 2(b) and (c) are the corresponding range-Doppler maps with the receiver matching the signal transmitted from radar A and radar B, respectively. It is evident that cross-radar interference maintains the Doppler frequency information whereas the fast-time (range) information is missing and the cross-radar interference spreads over the entire fast-time width (range). As a result, it is seen that, while cross-radar interference exists, the range and Doppler of the target can be clearly identified in this case. The average SNR (the ratio between the signal power and the average noise power over all range cells) is $36.7 \mathrm{~dB}$, and the SNR evaluated at the target range cell is $40.3 \mathrm{~dB}$. The SIR is $20.2 \mathrm{~dB}$, which is very close to the analytic result.

Fig. 3 shows the range-Doppler maps as a result of five iterations of cross-radar interference cancellation. In this plot, the average SNR and the cell SNR in the auto-radar reception mode remain at the same values of $36.7 \mathrm{~dB}$ and $40.3 \mathrm{~dB}$, respectively. In the cross-radar reception mode, the average SINR is also $36.7 \mathrm{~dB}$, and the average power of the residual error due to cross-radar interference is $7.5 \mathrm{~dB}$ lower than that of the original noise. This confirms that substantial interference mitigation has been achieved without compromising the target signal. 


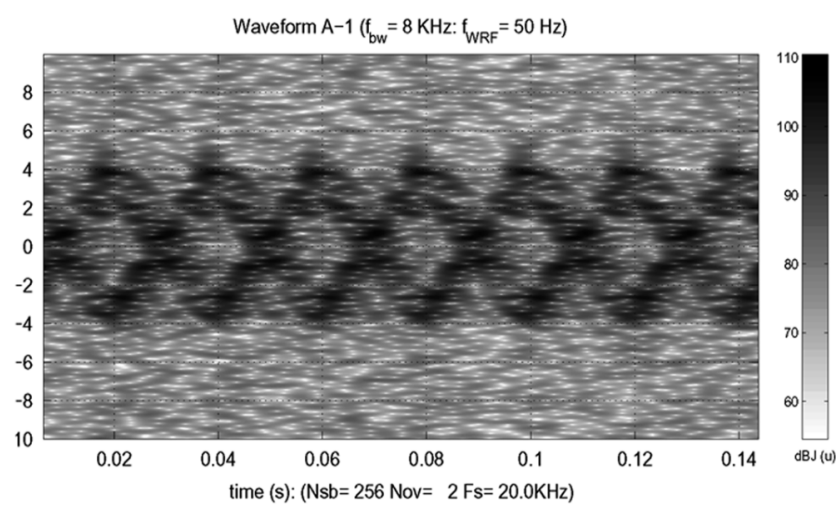

(a)

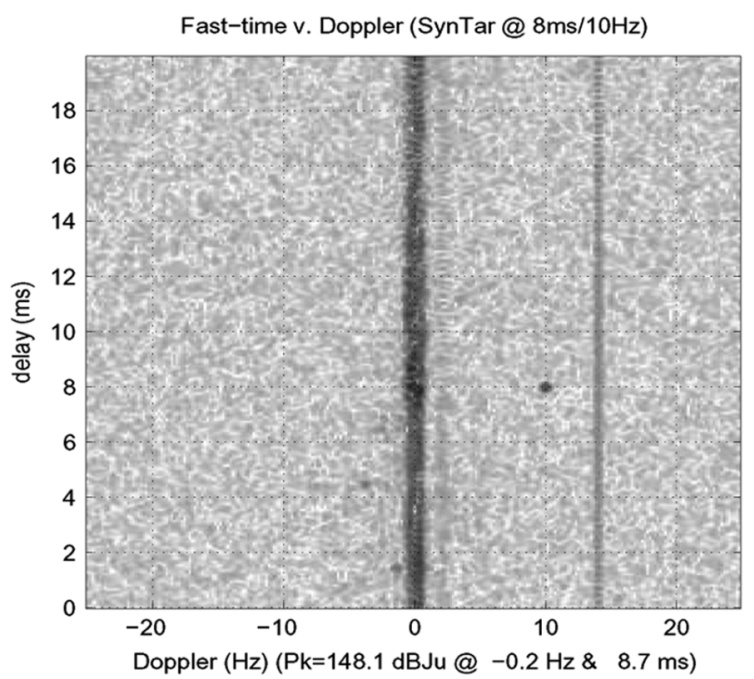

(b)

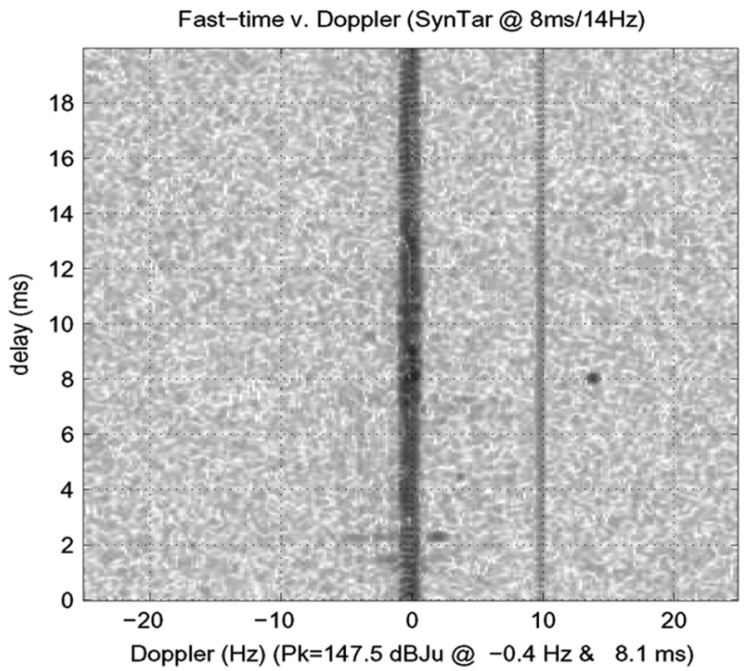

(c)

Fig. 2. Dual radar operation results without interference cancellation. (a) Timefrequency signature of the received signal, (b) Range-Doppler map matched to radar A's waveform, and (c) Range-Doppler map matched to radar B' waveform.

\section{CONCLUSION}

We have considered a concurrent operation of two OTHR systems for improved estimation of target maneuvering. A new method for cross-radar interference mitigation was developed

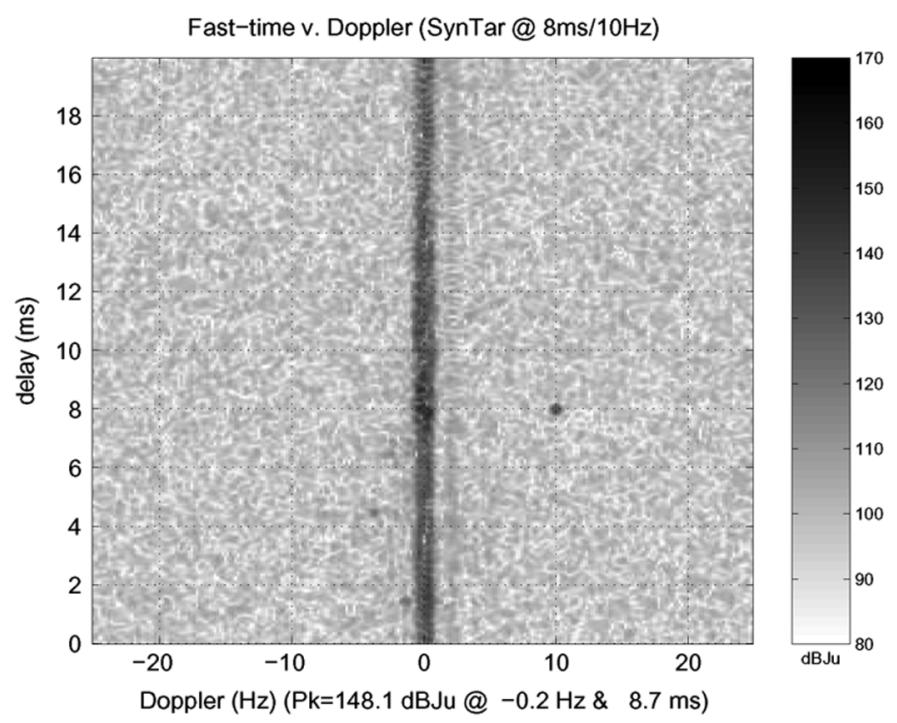

(a)

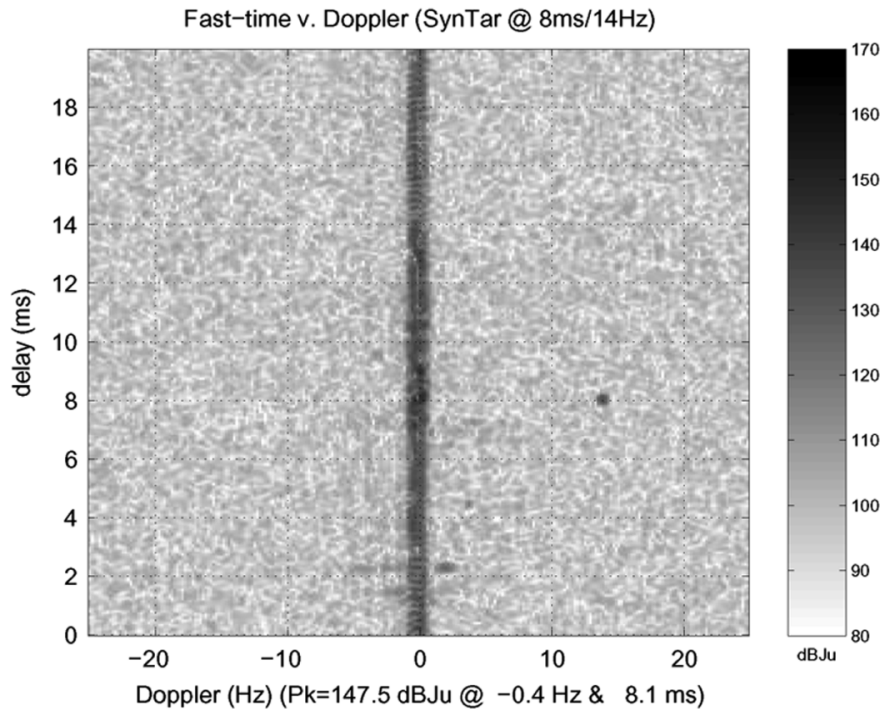

(b)

Fig. 3. Range-Doppler maps with interference cancellation. (a) Matched to radar A's waveform and (b) matched to radar B's waveform.

and its performance was analyzed. The usefulness of the proposed method was demonstrated by using measured clutter data and a synthetic test target. It was shown that applying crossradar interference cancellation techniques, such as those presented in this paper, can substantially suppress cross-radar interference without compromising the auto-radar responses, leading to enhanced target detection and characterization.

\section{ACKNOWLEDGMENT}

The authors would like to thank the insightful discussion by Dr. G. Wang. They also wish to thank the anonymous reviewers for their helpful comments on earlier versions of this paper.

\section{REFERENCES}

[1] J. M. Headrick and M. I. Skolnik, "Over-the-horizon radar in the HF band," Proc. IEEE, vol. 62, pp. 664-673, 1974.

[2] L. F. McNamara, The Ionosphere, Communications, Surveillance, and Direction Finding. Melbourne, FL: Krieger, 1991. 
[3] G. D. McNeal, "The high-frequency environment at the ROTHR Amchitka radar site," Radio Sci., vol. 30, pp. 739-746, May-June 1995.

[4] T. M. Georges and J. A. Harlan, "New horizons for over-the-horizon radar?," IEEE Antennas Propagat. Mag., vol. 36, no. 4, pp. 14-24, Aug. 1994.

[5] Y. Zhang, G. J. Frazer, and M. G. Amin, "Simultaneous operation of two over-the-horizon radars," in Proc. SPIE Advanced Signal Processing Algorithms, Architectures, and Implementations XIV, Denver, CO, Aug. 2004, pp. 313-324.

[6] J. R. Barnum and E. E. Simpson, "Over-the-horizon radar sensitivity enhancement by impulse noise excision," in Proc. IEEE Nat. Radar Conf., 1997, pp. 252-256.

[7] M. Turley, "Impulse noise rejection in HF radar using a linear prediction technique," in Proc. IEEE Int. Conf. Radar, 2003, pp. 358-362.

[8] G. Wang, X.-G. Xia, B. T. Root, V. C. Chen, Y. Zhang, and M. G. Amin, "Manoeuvring target detection in over-the-horizon radar using adaptive clutter rejection and adaptive chirplet transform," Proc. Inst. Elect. Eng., Radar, Sonar, Navig., vol. 150, no. 4, pp. 292-298, Aug. 2003.

[9] Y. Zhang, M. G. Amin, and G. J. Frazer, "High-resolution time-frequency distributions for manoeuvring target detection in over-the-horizon radars," Proc. Inst. Elect. Eng., Radar, Sonar, Navig., vol. 150, no. 4, pp. 299-304, Aug. 2003.

[10] X. Guo, H. Sun, and T. S. Yeo, "Transient interference excision in overthe-horizon radar using adaptive time-frequency analysis," IEEE Trans. Geosci. Remote Sensing, vol. 43, no. 4, pp. 722-725, Apr. 2005.

[11] D. C. Maori and J. H. G. Ender, "Performance analysis of multistatic configurations for spaceborne GMTI based on the auxiliary beam approach," Proc. Inst. Elect. Eng., Radar, Sonar, Navig., vol. 153, no. 2, pp. 96-103, Apr. 2006.

[12] S. D. Blunt and K. Gerlach, "Multistatic adaptive pulse compression," IEEE Trans. Aerosp. Electron. Syst., vol. 42, no. 3, pp. 891-902, Jul. 2006.

[13] J. Krolik, Target Localization and Track Association for Over-TheHorizon Radar With a Statistical Ionospheric Model Tech. Rep., Jul. 1999.

[14] D. E. Barrick, FM/CW radar signals and Digital Processing Tech. Rep. ERL283-WPL26, Jul. 1973, Nat. Ocean. Atmos. Admin.

[15] U. Madhow and M. Honig, "MMSE interference suppression for directsequence spread spectrum CDMA," IEEE Trans. Commun., vol. 42, pp. 3178-3188, Dec. 1994.

[16] S. Verdu, Multiuser Detection. Cambridge, U.K.: Cambridge Univ. Press, 1998

[17] X. Wang and H. V. Poor, "Space-time multiuser detection in multipath CDMA channels," IEEE Trans. Signal Processing, vol. 47, no. 9, pp. 2356-2374, Sep. 1999.

[18] J. Hogbom, "Aperture synthesis with a nonregular distribution of interferometer baselines," Astrophys. J. Suppl. Ser., vol. 15, pp. 417-426, 1974.

[19] U. J. Schartz, "Mathematical-statistical description of the iterative beam removing technique," Astron. Astrophys., vol. 65, pp. 345-356, 1978.

[20] Y. I. Abramovich, "A recursive method for the resolution of wideband signals with substantially different intensities," Radio Eng. Electron. Phys., vol. 23, no. 8, pp. 66-70, 1978.

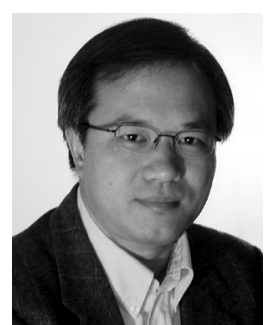

Yimin Zhang (SM'01) received the Ph.D. degree from the University of Tsukuba, Tsukuba, Japan, in 1988.

He joined the faculty of the Department of Radio Engineering, Southeast University, Nanjing, China, in 1988. He served as a Technical Manager at the Communication Laboratory Japan, Kawasaki, Japan, from 1995 to 1997, and was a Visiting Researcher at ATR Adaptive Communications Research Laboratories, Kyoto, Japan, from 1997 to 1998 . Since 1998, he has been with the Villanova University,
Villanova, PA, where he is currently a Research Associate Professor with the Center for Advanced Communications. His research interests lie in the area of statistical signal and array processing for communications and radar applications, including space-time adaptive processing, wireless networks, MIMO systems, cooperative diversity, blind signal processing, digital mobile communications, time-frequency analysis, source localization and tracking.

Dr. Zhang serves as an Associate Editor for IEEE SignAl PROCESSING LETTERS.

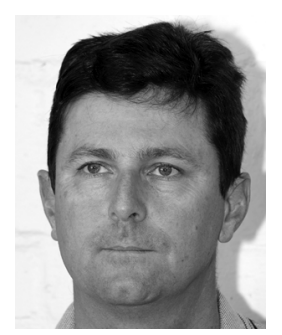

Gordon Frazer has been researching aspects of over-the-horizon radar (OTHR) since 1990. This includes extensive experience with several skywave and surface-wave radar systems including the JORN OTHR system, the Iluka I and II and SECAR surface-wave radar systems, and the Dundee and SkyLOS systems.

He is presently in charge of the High Frequency Radar Branch, ISR Division, Defence Science and Technology Organisation with responsibility for research programs in all facets of over-the-horizon radar. His personal research interests are in radar design, signal analysis and array signal processing.

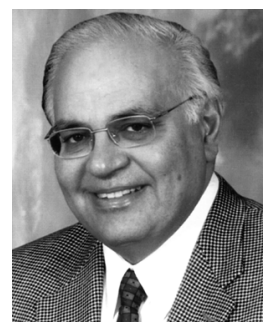

Moeness Amin (F'00) received the Ph.D. degree in 1984 from University of Colorado, Boulder.

He has been on the Faculty of Villanova University, Villanova, PA, since 1985, where is now a Professor in the Department of Electrical and Computer Engineering and the Director of the Center for Advanced Communications. He has over 300 publications in the areas of wireless communications, timefrequency analysis, smart antennas, interference cancellation in broadband communication platforms, direction finding, over the horizon radar, radar imaging, and channel equalizations.

Dr. Amin is a fellow of the International Society of Optical Engineers; Recipient of the IEEE Third Millennium Medal; Distinguished Lecturer of the IEEE Signal Processing Society for 2003; Member of the Franklin Institute Committee on Science and the Arts; Recipient of the 1997 Villanova University Outstanding Faculty Research Award; and Recipient of the 1997 IEEE Philadelphia Section Service Award. He was the Technical Chair of the 2nd IEEE International Symposium on Signal Processing and Information Technology, Morocco, 2002; the General and Organization Chair of the IEEE Workshop on Statistical Signal and Array Processing, Pennsylvania, 2000; and the General and Organization Chair of the IEEE International Symposium on Time-Frequency and Time-Scale Analysis, Pennsylvania, 1994. He was an Associate Editor of the IEEE TRANSACTIONS ON Signal PROCESSING from 1996 to 1998. He was a member of the IEEE Signal Processing Society Technical Committee on Signal Processing for Communications during 1998-2002 and was a Member of the IEEE Signal Processing Society Technical Committee on Statistical Signal and Array Processing during 1995-1997. 